\title{
Does Anesthesia Induction during Electrophysiologic Studies Induce Tachycardia in Pediatric Patients
}

\section{Manoj Gupta $^{1}$, Jill Shivapour ${ }^{1}$, Ann Lawrence ${ }^{2}$ and Christopher Snyder ${ }^{1 *}$}

${ }^{1}$ UH Division of Pediatric Cardiology, Rainbow Babies and Children's Hospital, Case Medical Center, Cleveland, Ohio, USA

${ }^{2}$ UH Division of Pediatric Anesthesia, Rainbow Babies and Children's Hospital, Case Medical Center, Cleveland, Ohio, USA

"Corresponding author: Christopher Snyder, Director, Division of Pediatric Cardiology, Associate Professor of Pediatrics, Case Western Reserve University School of Medicine, Rainbow Babies and Children's Hospital, USA, Tel: 216-844-8529; Fax: 216-844-5478; E-mail: Christopher.snyder@uhhospitals.org

Rec date: June 22, 2015; Acc date: July 20, 2016; Pub date: July 25, 2016

Copyright: ( 2016 Gupta M, et al. This is an open-access article distributed under the terms of the Creative Commons Attribution License, which permits unrestricted use, distribution, and reproduction in any medium, provided the original author and source are credited.

\section{Abstract}

Background: Anesthesia is essential to facilitate the majority of pediatric electrophysiology studies (EPS); however, a risk of an arrhythmia exists during its induction. The purpose of this study was to determine the incidence of arrhythmia during anesthesia induction in pediatric EPS.
\end{abstract}

Methods: IRB approved retrospective review of pediatric EPS from 1/99 to 1/14. Inclusion criteria: Age $\leq 21$ years and comprehensive EPS under general anesthesia. Data collected: demographics, EPS results, and anesthesia records. Patients were grouped based upon route of anesthesia administration: Intravenous (IV) agents (propofol, ketamine, and kentanyl) and Inhalational $(\mathrm{IH})$ agents (sevoflurane, isoflurane and desflurane).

Sinus Tachycardia (STACH) was defined as variable heart rates of $>140$ and $<180$ beats per minute (bpm) originating from sino-atrial node with a duration of $\geq 5$ minutes. Supraventricular Tachycardia (SVT) was defined as an abnormal heart rhythm arising due to abnormal electrical activity of the heart, originating proximal to the bundle of His, with sustained rates $\geq 180 \mathrm{bpm}$.

Results: Inclusion criteria were met by 378 patients, $57 \%$ male, median age $14 \pm 4$ years, with IV induction utilized in $275(72 \%)$ patients. During induction, $39(10 \%)$ developed tachycardia: 38 STACH and 1 SVT. STACH was more common with IH anesthesia when compared to IV $(p<0.0001)$. Patients with Wolff-Parkinson-White (WPW), irrespective of anesthesia type, had twice the risk of developing STACH, 17/114 vs. 21/264 of others ( $p=$ $0.02)$.

Conclusion: Sinus Tachycardia occurred in $10 \%$ of pediatric electrophysiology cases performed under anesthesia. It was seen more frequently when IH agents were used compared to IV. Patients with WPW have double the risk of developing sinus tachycardia during anesthesia induction when compared to patients with other forms of SVT.

Keywords: Supraventricular tachycardia; Wolff-Parkinson-White syndrome; Pediatric electrophysiology study; Route of Anesthesia induction; Atrioventricular Nodal Reentrant Tachycardia

\section{Introduction}

An Electrophysiology study (EP study) is a specialized intra-cardiac procedure utilizing catheters to evaluate the cardiac conduction system. Due to the highly technical nature of these studies and the fact that they are often performed on children and adolescents, it is important to perform these procedures while the patient is comfortable and/or asleep. These studies can be performed under sedation only in older adolescents if the patient is cooperative. The use of general anesthesia to allow pediatric patients to undergo a comfortable electrophysiology study (EPS) is becoming the standard of care. The use of anesthetic agents during these pediatric procedures has been well documented and its use is rarely associated with rare lifethreatening cardiovascular adverse events [1]. The cardiovascular side effects associated with the utilization of anesthetic agents include cardiac arrest, desaturation, hypotension, bradycardia/tachycardia, and changes in cardiomyocyte repolarization time (QT prolongation) resulting in arrhythmias [1-8].

Inhalational anesthetic agents such as sevoflurane, isoflurane and desflurane have been utilized by pediatric anesthesiologists during operating room cases and have reported occurrences of arrhythmias [4,8-11]. The initiating factors for these arrhythmias consist of transient electrolyte imbalances, hypotension, hypertension and fluctuation in core body temperature [3,4]. Additional cardiac effects associated with the use of these agents includes decrease in effective refractory period (ERP), changes in the heart rate and the ventricular activation time have been reported [2-4]. These agents also affect the myocardial potassium channels leading to prolongation of QTc interval and subsequent arrhythmias [1-4].

Pediatric patients undergo EPS for diagnosis and treatment of their arrhythmias. There is an assumed risk of arrhythmia occurrence during anesthesia induction, but the incidence and nature of these arrhythmias in pediatric patients undergoing these procedures is unknown. To date, no studies have investigated the risk of arrhythmias during anesthesia induction for EPS. Understanding when and if 
Page 2 of 4

arrhythmias occur during induction may lead to identification of less arrhythmogenic agents that could be utilized in the future.

The primary objectives of this study were to determine the incidence and type of arrhythmias during induction of anesthesia in pediatric patients undergoing EPS and if they differ when utilizing different anesthetic agents and modes of anesthesia.

\section{Material and Methods}

Institutional review board (IRB) approved retrospective chart review was performed for all the patients who had an EPS from 01/99 to $01 / 14$. The data collected included demographics, indications for EP study, route of anesthesia administration, medications used for induction, and occurrence and type of arrhythmia during induction. Routine monitoring during anesthesia induction included continuous electrocardiography (ECG), blood pressure monitoring, peripheral pulse oximetry and respiratory rate monitoring. Intermittent positive pressure ventilation was used to maintain an end-tidal carbon dioxide (ETCO2) in the normal range of $40-48 \mathrm{mmHg}$ when available. The endotracheal intubation was facilitated by rocuronium or vecuronium.

Anesthesia and EP reports were recorded. The patients were then divided into 2 groups based upon mode of anesthesia induction either (1) Inhalational anesthesia (IH) or (2) intravenous anesthesia (IV). The inclusion criteria were: age $<21$ years, EPS performed under general anesthesia. Those patients with congenital heart disease were excluded. The frequency and type of arrhythmias that occurred during anesthesia induction were documented. All data were collected and analyzed using the SAS software (SAS software version 9.3; SAS, Campus Drive, Cary, NC). The differences between variables were assessed using Chi-Square test, and Fisher exact test and the differences were considered significant at a $\mathrm{p}$ valve of $<0.05$. Patient characteristics (including gender, age, weight and height), type of anesthesia induction and types of arrhythmia between the two groups were compared. For the purposes of this study, all WPW patients and those with ventricular pre-excitation were categorized as WPW.
For the purpose of this study, General anesthesia was defined as general insensibility to pain and other sensations induced by certain interventions and drugs to permit the performance of surgery or painful procedures. Inhalational induction $(\mathrm{IH})$ was defined as induction of anesthesia through mask using inhaled anesthetics, and examples include isoflurane, sevoflurane, desflurane and nitrous oxide (N2O). Intravenous induction (IV) was defined as induction of anesthesia using intravenous agents, and examples include propofol, fentanyl, midazolam, lorazepam and ketamine. Supraventricular tachycardia (SVT) was defined as a tachycardia that originates proximal to the bundle of His with consistent heart rate of $>180 \mathrm{bpm}$. Sinus tachycardia (STACH) was defined as persistent and variable heart rates $>140$ and $<180 \mathrm{bpm}$ originating from SA node. Sinus Bradycardia was defined as sinus rhythm originating from SA node with a persistent heart rate of less than $50 \mathrm{bpm}$. Hypotension was defined as a systolic blood pressure $<70 \mathrm{mmHg}$ at any time during anesthesia induction. The anesthesia induction time was defined as the time from initiation of anesthesia induction to the time of first intravenous access attempt for EPS. The maintenance phase was defined as the point when the drugs used to initiate the anesthetic are beginning to wear off and the anesthesia is then maintained with maintenance agent, typically an inhaled or intravenous anesthetic. Isoflurane was used for induction in earlier part of this study but after 2005 , it was mainly used for anesthesia's maintenance.

\section{Results}

The data search revealed 408 pediatric patients that underwent EPS under general anesthesia, of which 378 (93\%) met inclusion criteria. The median age at the time of the EP study was 14 years (mean $13.5 \pm$ 4 years) with 217 (57\%) being males. The most common indications for the EPS were concealed pathway (34\%), WPW (30\%), and AVNRT (26\%) (Table 1).

\begin{tabular}{|c|c|c|}
\hline Characteristics and demographics & Number & Percent \\
\hline Age in years $( \pm S D)$ & $13.5( \pm 4)$ & \\
\hline Gender(Male) & 217 & $57 \%$ \\
\hline \multicolumn{3}{|l|}{ Route of anesthesia induction } \\
\hline Inhalational anesthesia & 103 & $27 \%$ \\
\hline Intravenous anesthesia & 275 & $73 \%$ \\
\hline \multicolumn{3}{|l|}{ Indications for EP study } \\
\hline WPW & 114 & $30 \%$ \\
\hline AVRT/Concealed pathway & 129 & $34 \%$ \\
\hline AVNRT & 99 & $26 \%$ \\
\hline Others & 36 & $9 \%$ \\
\hline
\end{tabular}

Table 1: Common indications for the EPS.

The patients that met inclusion criteria were then divided into 2 groups based on route of anesthesia induction: IV vs. IH. IV anesthetic

agents were administered to 275 (73\%) patients while 103 received $\mathrm{IH}$ agents. 
No statistically significant differences existed between the groups with respect to other demographics.

Tachycardia was recorded in 39 patients (10\%) during anesthesia induction, with STACH accounting for 38 and SVT in one patient. When tachycardia was analyzed based on route of anesthesia administration, a significantly higher number of patients that had $\mathrm{IH}$ anesthesia, 22/103 (21\%) developed tachycardia compared to $16 / 275$ $(6 \%)$ that had IV $[\mathrm{p}<0.0001]$ (Table 2 ). In the IH group, 1 patient developed SVT as well. Upon further analysis of those with STACH, it was noted to occur more frequently in male patients (13\%) compared to female patients $(5 \%)[p=0.0237]$.

\begin{tabular}{|l|l|l|l|}
\hline $\begin{array}{l}\text { Arrhythmias during } \\
\text { anesthesia } \\
\text { induction }\end{array}$ & $\begin{array}{l}\text { Intravenous } \\
\text { anesthesia }\end{array}$ & $\begin{array}{l}\text { Inhalational } \\
\text { anesthesia }\end{array}$ & p value \\
\hline Sinus tachycardia & 16 & $2200 \%$ & $<0.0001$ \\
\hline SVT & 0 & 1 & \\
\hline SVT: Supraventricular tachycardia &
\end{tabular}

Table 2: Arrhythmias during anesthesia induction.

Another group that had relatively frequent episodes of tachycardia during anesthesia induction was those with WPW. In this cohort of patients, 17/114 (15\%) had STACH and 1 had SVT (Table 3). In those with concealed pathway 18/129 (14\%) developed STACH and only 2/99 (2\%) AVNRT patients developed STACH during anesthesia induction $[\mathrm{p}=\mathrm{NS}]$.

\begin{tabular}{|l|l|l|}
\hline Indications for EP study & $\begin{array}{l}\text { SVT } \\
\text { anesthesia } \\
\text { induction }\end{array}$ & $\begin{array}{l}\text { Sinus tachycardia } \\
\text { during anesthesia } \\
\text { induction }\end{array}$ \\
\hline WPW & $100 \%$ & $17(114)$ \\
\hline Concealed pathway & $0 \%$ & $18(129)$ \\
\hline AVNRT & 0 & $2(99)$ \\
\hline Others & $0 \%$ & $1(36)$ \\
\hline
\end{tabular}

Table 3: Indications for EP study.

\section{Discussion}

Due to the complexity of a pediatric EPS and for the safety and comfort of children during these procedures, the use of pediatric anesthesia is becoming more widespread. Instead of utilizing any anesthetic agent for pediatric EPS, the optimal anesthetic agent should have minimal effect on cardiac conduction tissue specifically in both the normal atrioventricular conduction system and in the accessory pathway(s) and that anesthetic agents should adequately suppress the sympathetic responses to induction and stimulation to allow predictable progression of procedure.

Multiple anesthetic agents have been reported that would allow rapid titrations to prevent unwanted responses and make patients comfortable. Much has been published about potential side effects of these anesthetic agents on cardiac and accessory pathway conduction during EPS [7-12], but little is published about the potential cardiovascular side effects of anesthetic agents during induction and their propensity to induce any type of tachycardia during anesthesia induction.
This study illustrated the incidence of tachycardia during anesthesia induction. The patients receiving $\mathrm{IH}$ anesthesia during induction experienced significantly more sinus tachycardia (21\%) when compared to those that received IV anesthesia agents $(6 \%)[\mathrm{p}<0.0001]$. This may be due to a higher incidence of hemodynamic disturbances like hypotension, peripheral vasodilatation, a decrease in cardiac output due to depressed myocardial contractility leading to hypotension, or secondary to cardiac conduction tissue sensitization to IH agents. In addition, isoflurane and desflurane are also known to produce a dose dependent increase in heart rate [7-12].

Induction using an IV anesthetic agent caused significantly less tachycardia when compared to inhalational agents $[p<0.0001]$; suggesting potentially less arrhythmogenic effects of propofol and ketamine on the electrophysiological properties of normal and accessory pathway conducting system. A number of studies have demonstrated less cardiovascular side effects of propofol on conduction properties of normal and accessory pathways in patients with WPW syndrome during adult EP studies [7-12]. One study showed successful use of propofol for EP study for treatment of tachyarrhythmias except for atrial ectopic tachycardia $[5,6]$. Halogenated anesthetic agents (sevoflurane, isoflurane and desflurane) have resulted in prolongation of the repolarization time of the heart leading to QTc prolongation and induction of arrhythmias in various studies $[4,8,11]$. Isoflurane has also been reported to increase the antegrade accessory pathway effective refractory period in children with WPW [11]; however, effects on the AV nodal conduction time were not reported $[8,11]$.

Based on the results from this study, SVT occurrence during anesthesia induction is rare (1/376). The use of $\mathrm{IH}$ anesthetic agents increased the occurrence of STACH during anesthesia induction, and therefore, these agents should be used with caution in patients where tachycardia can affect the hemodynamic stability or ability to perform the EP study. The reasons may be secondary to a decrease in peripheral vascular resistance, dehydration, or direct effect of the inhalational agent on the cardiac conduction tissue. Further molecular and basic science studies are needed to better define the direct effects of inhalational agents on sino-atrial nodal tissue.

\section{Conclusion}

Sinus tachycardia (STACH) is seen in $10 \%$ of pediatric patients undergoing EP study during anesthesia induction. SVT occurrence during anesthesia induction is rare, occurring in less than $1 \%$ of all cases. Inhalational anesthesia use has more risk of developing sinus tachycardia during induction compared to Intravenous agents. Those patients with WPW have double the risk of developing sinus tachycardia when compared to non-WPW patients. Male patients undergoing EP studies under general anesthesia have a significantly higher risk of developing STACH.

\section{References}

1. Owczuk R, Wujtewicz MA, Zienciuk A, Lasinska-Kowara M, Piankowski A, et al. (2012) The influence of anesthesia on cardiac repolarization. Minerva Anestesiol 78: 483-495.

2. Kuner J, Enescu V, Utsu F, Boszormenyi E, Bernstein H, et al. (1967) Cardiac Arrhythmias during Anesthesia. Chest 52: 580-587.

3. Cravero JP, Beach ML, Blike GT, Gallagher SM, Hertzog JH, et al. (2009) The Incidence and Nature of Adverse Events During Pediatric Sedation/ Anesthesia With Propofol for Procedures Outside the Operating Room: A 
Citation: Gupta M, Shivapour J, Lawrence A, Snyder C (2016) Does Anesthesia Induction during Electrophysiologic Studies Induce Tachycardia in Pediatric Patients. J Cardiovasc Dis Diagn 4: 250. doi:10.4172/2329-9517.1000250

Page 4 of 4

Report From the Pediatric Sedation Research Consortium. Anesthesia \& Analgesia 108: 795-804.

4. Deutsch N, Hantler CB, Tait AR, Uprichard A, Schork MA, et al. (1990) Suppression of Ventricular Arrhythmias by Volatile Anesthetics in a Canine Model of Chronic Myocardial Infarction. Anesthesiology 72: 1012-1021.

5. Lai LP, Lin JI, Wu MH, Wang MJ, Huang CH, et al. (1999) Usefulness of Intravenous Propofol Anesthesia for Radiofrequency Catheter Ablation in Patients with Tachyarrhythmias: Infeasibility for Pediatric Patients with Ectopic Atrial Tachycardia. Pacing and Clinical Electrophysiology 22: $1358-1364$.

6. Lavoie J, Walsh EP, Burrows FA, Laussen P, Lulu JA, et al. (1995) Effects of Propofol or Isoflurane Anesthesia on Cardiac Conduction in Children Undergoing Radiofrequency Catheter Ablation for Tachydyshythmias. Anesthesiology 82: 884-887.

7. Liu Q, Kong AL, Chen R, Qian C, Liu SW, et al. (2011) Propofol and arrhythmias: two sides of the coin. Acta Pharmacologica Sinica 32: 817-823.

8. Sharpe MD, Dobkowski WB, Murkin JM, Klein G, Guiraudon G, et al. (1994) The Electrophysiologic Effects of Volatile Anesthetics and Sufentanil on the Normal Atrioventricular Conduction System and
Accessory Pathways in Wolff-Parkinson-White Syndrome. Anesthesiology 80: 63-70.

9. Niksch A, Liberman L, Clapcich A, Schwarzenberger JC, Silver ES, et al (2010) Effects of Remifentanil on Cardiac Electrophysiologic Properties in Children Undergoing Catheter Ablation of Supraventricular Tachycardia. Pediatr Cardiol 31: 1079-1082.

10. Sharpe MD, Dobkowski WB, Murkin JM, Klein G, Yee R (1995) Propofol Has No Direct Effect on Sinoatrial Node Function or on Normal Atrioventricular and Accessory Pathway Conduction in WolffParkinson-White Syndrome during Alfentanil/Midazolam Anesthesia; Anesthesiology 82: 888-895.

11. Chang RK, Stevenson WG, Wetzel GT, Shannon K, Baum VC, et al. (1996) Effects of Isoflurane on Electrophysiological Measurements in Children with the Wolff-Parkinson-White Syndrome; PACE 19: 1082-1088.

12. Sharpe MD, Dobkowski WB, Murkin JM, Klein G, Guiraudon G, et al. (1992) Alfentanil-midazolam anaesthesia has no electrophysiological effects upon the normal conduction system or accessory pathways in patients with Wolff-Parkinson-White syndrome Can J Anaesth 39: 816-821. 